\title{
Combined nerve conduction index in diabetic polyneuropathy
}

\author{
Índice combinado de condução nervosa na polineuropatia diabética \\ Carlos Otto Heise, Flavia Costa Nunes Machado, Simone Consuelo de Amorim, Sonia Maria de Toledo
}

\begin{abstract}
Diabetic polyneuropathy can be confirmed by nerve conduction studies. The data can be analyzed in the form of a combined index instead of individual parameters. Methods: The combined index included five parameters of nerve conduction studies commonly used for evaluation of polyneuropathies. We evaluated sensitivity in 100 diabetic patients with suspected polyneuropathy, and specificity in 200 non-diabetic patients with suspected lumbosacral radiculopathy. All results were expressed in number of standard deviations (SD). Results: The sensitivity of the combined index was 81 or $74 \%$, and specificity was 97 or $98 \%$, using respectively -2.0 or -2.5 SD as cutoff. The range of sensitivity of the other parameters was $57-65 \%$ or $48-56 \%$, and specificity range was $96-98 \%$ or $98-100 \%$, using the same criteria. Discussion: The combined index had higher sensitivity and equivalent specificity compared to isolated parameters.
\end{abstract}

Key words: diabetes mellitus, polyneuropathies, neural conduction, electrodiagnosis.

\section{RESUMO}

A polineuropatia diabética pode ser confirmada por estudos de condução nervosa. Os dados podem ser analisados por um índice combinado ao invés de parâmetros isolados. Métodos: 0 índice combinado incluiu cinco parâmetros usados rotineiramente na avaliação de polineuropatias. Avaliamos a sensibilidade em 100 pacientes diabéticos com suspeita de polineuropatia e a especificidade em 200 pacientes não diabéticos com suspeita de radiculopatia lombossacral. Todos os resultados foram expressos em número de desvios-padrão (DP). Resultados: A sensibilidade do índice combinado foi 81 ou 74\%, e a especificidade foi 97 ou 98\%, usando respectivamente -2,0 ou -2,5 DP como ponto de corte. A sensibilidade dos parâmetros isolados oscilou entre $57-65 \%$ ou 48-56\%, e a especificidade foi 96-98\% ou 98-100\%, usando os mesmos critérios. Discussão: 0 índice combinado apresentou melhor sensibilidade e especificidade equivalente aos parâmetros isolados.

Palavras-Chave: diabetes mellitus, polineuropatias, condução nervosa, eletrodiagnóstico.

Nerve conduction studies are commonly used to confirm diabetic polyneuropathy ${ }^{1}$ and should be included as part of the definition of distal polyneuropathy for clinical research². They provide objective data and are generally more accurate than clinical evaluation ${ }^{3}$. Nerve conduction studies are composed of several tests, including motor and sensory responses. Each test is performed in few individual nerves and provides two to five different parameters. In standard neurophysiological evaluation of the lower limbs, more than twenty different parameters are evaluated, and each one has its own normative data. Each parameter is usually defined as "normal" or "abnormal". This is a simplistic interpretation, since borderline values may be encountered. Moreover, normal values are influenced by factors such as age and height ${ }^{4-6}$, thus differing among patients.

A better way to express each parameter is providing the Z-score, which is the number of standard deviations (SD) from the mean expected value $e^{4}$ Mean expected values can be adjusted to biological variables such as age and height. Results that are more than 2 SD away from the mean expected value are considered abnormal. This classic cutoff point provides a theoretical specificity of $97.7 \%$. If a higher specificity is desired, a higher and more conservative cutoff point may be used, such as 2.5 or $3.0 \mathrm{SD}$. Z-scores provide information regarding "how normal" or "how abnormal" is the parameter in a continuous form. They are also more intuitive to interpret and do not require the knowledge of each normative value. The parameter must have a normal distribution in control subjects to be expressed as a Z-score. This is not the case for amplitudes of the potentials recorded in nerve conduction studies, but they can be transformed into a logarithm scale to fit a normal distribution ${ }^{4,7}$.

Another advantage of Z-scores is that they can be combined. Since all parameters are provided in the same way, 
they can be averaged. This provides a unique value representing the whole neurophysiological exam. When averaging several Z-scores from a normal subject, random experimental errors from each parameter are canceled and the averaged value tends to zero ${ }^{4,7}$. This means that the SD of the averaged Z-score is smaller than it should be, so it must be also analyzed in normal controls to establish its distribution ${ }^{7}$. After that, it can also be expressed as a single combined Z-score index and interpreted in the same way.

The utilization of a single combined index has many advantages over the use of several isolated parameters. The most obvious one is that it is easier to interpret. Besides that, several subnormal parameters may become abnormal when analyzed together. On the other hand, a single abnormal parameter obtained because of a technical error would be compensated by the remaining normal values. Theoretically, a combined index could enhance both sensitivity and specificity of nerve conduction studies ${ }^{8}$.

In this study, we compared sensitivity and specificity of five different parameters of nerve conduction study with a combined index to detect polyneuropathy in symptomatic diabetic patients.

\section{METHODS}

Regression equations to calculate Z-scores were obtained from a previous study in our institution using 125 control subjects with age varying from 18 to 86 years (mean $46)^{9}$. We analyzed five parameters in each lower limb (total of ten in each patient): sensory conduction velocity of the sural nerve (SCV-S), natural logarithm of the amplitude of the sensory action potential of the sural nerve (ASP-S) and superficial peroneal nerve (ASP-P), motor conduction velocity of the peroneal nerve (MCV-P) and F-M minimal latency of the tibial nerve (FW-T). The equation for Z-score calculation was: $Z=(V o-V e) / S D$, where $V o$ is the observed value of the parameter, $V e$ is the mean expected value corrected by age and height, and $S D$ is the standard deviation observed in the normative group. The expected value was obtained by regression analysis according to the general equation: $V e=b \cdot a^{2}+c \cdot a+d \cdot h+e$, where $a$ is age in years, $h$ is height in centimeters and $b, c, d$, and $e$ are experimental calculated coefficients. The coefficients and standard deviations of each parameter are listed in Table.

We retrospectively analyzed 100 consecutive diabetic patients referred to our diagnostic center for neurophysiological evaluation of clinically suspected diabetic polyneuropathy. All patients had glycosylated haemoglobin levels above $6.5 \mathrm{mg} \%$ and reported paresthesia or burning sensation in both feet. Patients older than 65 years of age or with other known cause for polyneuropathy were excluded. We also analyzed retrospectively 200 consecutive non-diabetic patients referred for suspected lumbosacral radiculopathy. All patients had lumbosacral pain radiating down to one leg and fasting glucose levels below $99 \mathrm{mg} / \mathrm{dL}$. Patients older than 65 years and those with bilateral symptoms were excluded. This study was approved by the ethics research committee of our institution.

Nerve conduction studies were performed according to our standard protocol using a Portable Keypoint equipment (Dantec, Skovlunde, Denmark). Skin temperature was kept above $30^{\circ} \mathrm{C}$. Sensory nerve action potentials of sural and superficial peroneal nerves were recorded using a bar electrode of $3 \mathrm{~cm}$, with fixed distance of $14 \mathrm{~cm}$ between the stimulating and recording sites. We measured ASP-S and ASP-P in microvolts, and SCV-S in meters per second. Motor conduction studies were recorded with adhesive electrodes (Dantec 9013L0452, Skovlunde, Denmark) located over usual recording sites. MCV-P was recorded between the ankle and the fibular head, and expressed in meters per second. We measured the shortest of 10 tibial $F$ waves latencies and subtracted the distal motor latency (M latency) to calculate FW-T in milliseconds.

All values were converted to Z-scores according to the equations in Table. FW-T was expressed in negative form, so that all negative Z-scores corresponded to subnormal values. We obtained ten Z-scores from each patient (five from each lower limb), calculated the mean Z-score and used the last equation in Table to obtain the combined index. All

Table. Equations for Z-score calculation.

\begin{tabular}{|c|c|c|c|c|c|}
\hline \multirow{2}{*}{$\begin{array}{l}\mathrm{Z}=(\mathrm{Vo}-\mathrm{Ve}) / \mathrm{SD} \\
\text { Parameter }\end{array}$} & \multicolumn{5}{|c|}{$V e=b \cdot a^{2}+c \cdot a+d \cdot h+e$} \\
\hline & $b$ & $\mathrm{C}$ & $d$ & e & SD \\
\hline ASP-S & -0.00029 & 0.0132 & -0.0133 & 5.029 & 0.418 \\
\hline SCV-S & -0.00025 & -0.0577 & -0.0944 & 69.40 & 3.994 \\
\hline ASP-P & -0.00029 & 0.0129 & -0.0172 & 5.206 & 0.517 \\
\hline MCV-P & -0.00025 & -0.0497 & -0.1154 & 71.66 & 2.761 \\
\hline FW-T & 0.00041 & 0.0010 & 0.2909 & -5.94 & $-2.772 *$ \\
\hline Combined Index & 0 & -0.0085 & -0.0077 & 1.743 & 0.524 \\
\hline
\end{tabular}

Z:Z-score;Vo: observed value; Ve: expected value; SD: standard deviation; a: age (years); h: height (centimeters); b, c, d, and e: experimental coefficients; ASP-S: natural logarithm of the amplitude of the sensory action potential of the sural nerve ( $\mu \mathrm{V})$; SCV-S: sensory conduction velocity of the sural nerve (m/s); ASP-P: natural logarithm of the amplitude of the sensory action potential of superficial peroneal nerve ( $\mu \mathrm{V})$; MCV-P: motor conduction velocity of the peroneal nerve $(\mathrm{m} / \mathrm{s})$; FW-T: F-M minimal latency of $10 \mathrm{~F}$ waves of the tibial nerve (ms). *Negative value of the standard deviation, so that negative Z-scores values refer to prolonged F waves. 
calculations were done using a Microsoft Excel spreadsheet (Microsoft Corporation, Redmond, USA). If any parameter were unobtainable, the mean was calculated from the remaining Z-scores. If all parameters were unobtainable, the Z-score was also considered unobtainable. We used two different cutoff levels to define abnormality: -2.0 and -2.5 SD. Unobtainable parameters were also considered abnormal.

We compared sensitivity of the combined index and of the five isolated parameters to detect polyneuropathy in the diabetic group of patients. We also compared specificity of the combined index and of the isolated parameters in the group of non-diabetic patients. The combined index and isolated parameters were compared through Chi-square test. Differences were considered statistically significant when $\mathrm{p}<0.05$.

\section{RESULTS}

The group of symptomatic diabetic patients was composed of 39 women and 61 men. Age ranged from 25 to 65 years (median 57). The non-diabetic group was composed of 113 women and 87 men, with age ranging from 21 to 65 years (median 47).

The sensitivity of the combined index was $81.0 \%$, when using 2 SD as cutoff point. Sensitivities of the other parameters were: ASP-S - 62.5\%; SCV-S - 57.0\%; ASP-P - 58.5\%; MCV-P - 64.5\%; and FW-T - 59.0\%. The combined index was more sensitive than any other isolated parameter $(\mathrm{p}<0.05)$. If any single abnormality was considered, the five parameters together yielded a sensitivity of $84.0 \%$, which was not significantly different from the combined index. Using the same cutoff point, the specificity of the combined index was
97.0\%. Specificities of the other parameters were: ASP-S 97.0\%; SCV-S - 97.0\%; ASP-P - 98.0\%; MCV-P - 96.3\%; and FW-T - 96.0\%. There was no significant difference in specificity between the combined index and the other parameters. If any single abnormality was considered, the five parameters together yielded a specificity of $81.0 \%$, which was less specific than the combined index $(\mathrm{p}<0.05)$.

The sensitivity of the combined index was $74.0 \%$, when using 2.5 SD as cutoff point. Sensitivities of the other parameters were: ASP-S - 56.0\%; SCV-S - 48.0\%; ASP-P - 52.5\%; MCV-P - 55.5\%; and FW-T - 53.0\%. The combined index was again more sensitive than any other isolated parameter $(\mathrm{p}<0.05)$. If any single abnormality was considered, the five parameters together yielded a sensitivity of $70.0 \%$, which was not significantly different from the combined index. Using the same cutoff point, the specificity of the combined index was 98.0\%. Specificities of the other parameters were: ASP-S - 98.0\%; SCV-S - 99.8\%; ASP-P - 98.8\%; MCV-P - 99.5\%; and FW-T - 99.0\%. There was no significant difference in specificity between the combined index and the other parameters. If any single abnormality was considered, the five parameters together yielded a specificity of $94.5 \%$, which was not significantly different from the combined index. Sensitivities of the combined index and of all isolated parameters with both cutoff points are shown in Fig 1, while specificities are shown in Fig 2.

\section{DISCUSSION}

This study showed that our combined index is more sensitive than isolated common nerve conduction parameters for diagnosis of clinically suspected diabetic polyneuropathy.

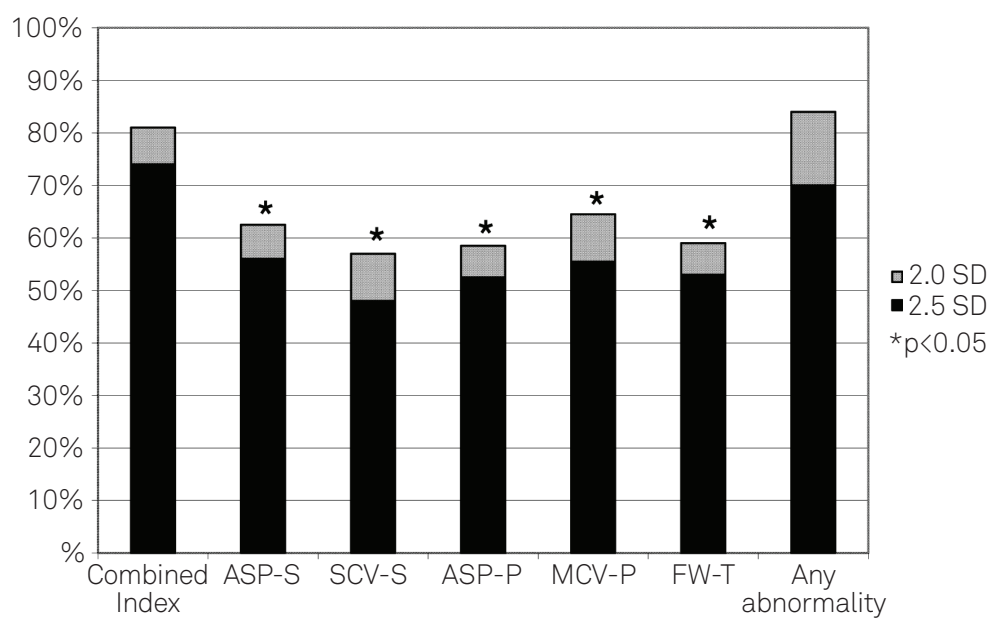

Fig 1. Sensitivity of the combined index compared to isolated parameters in 100 symptomatic diabetic patients. SD: standard deviation; ASP-S: natural logarithm of the amplitude of the sensory action potential of the sural nerve; SCV-S: sensory conduction velocity of the sural nerve; ASP-P: natural logarithm of the amplitude of the sensory action potential of the superficial peroneal nerve; MCV-P: motor conduction velocity of the peroneal nerve; FW-T: F-M minimal latency of the tibial nerve. 


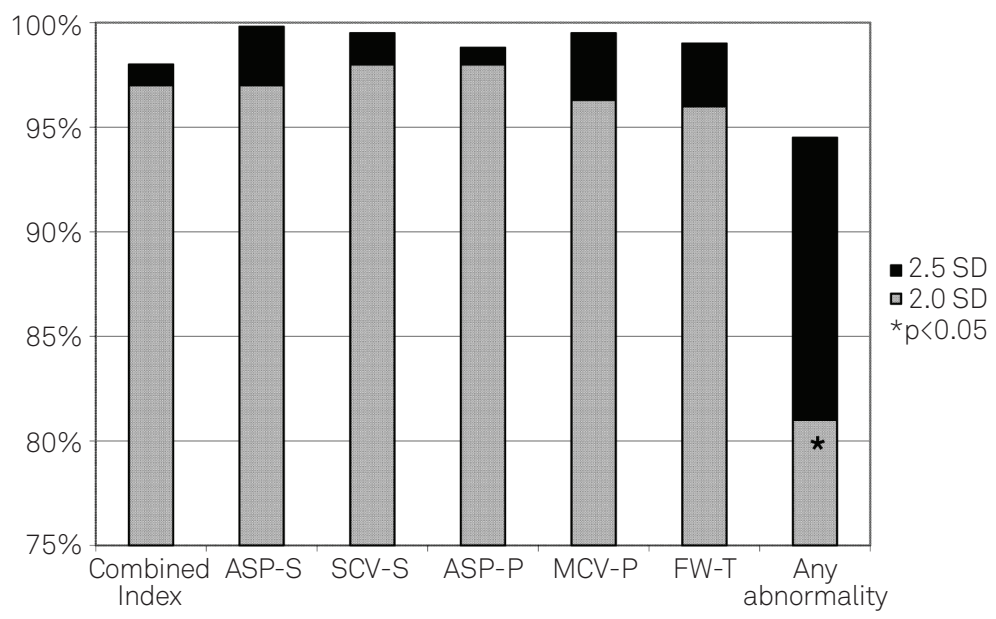

Fig 2. Specificity of the combined index compared to isolated parameters in 200 non-diabetic patients with suspected lumbosacral radiculopathy. SD: standard deviation; ASP-S: amplitude of the sensory potential of the sural nerve; SCV-S: sensory conduction velocity of the sural nerve; ASP-P: amplitude of the sensory potential of the superficial peroneal nerve; MCV-P: motor conduction velocity of the peroneal nerve; FW-T: F-M minimal latency of the tibial nerve.

If any single abnormality was considered, sensitivity of the combined index was similar to that of all parameters taken together; however, specificity of the combined index was higher. This effect was observed only if the cutoff point of 2 SD was used, but not with 2.5 SD. The probable cause for this discrepancy was insufficient power of the study; a larger number of patients would be required to demonstrate the same effect when using the 2.5 SD cutoff point.

How many different nerve conduction tests should be done to confirm diabetic polyneuropathy? It is logical that several tests would yield better sensitivity, but it also increases the chance of abnormal results with no clinical significance, thus reducing specificity. From the mathematical point of view, if we considered five independent tests with sensitivity of $60 \%$ and specificity of $97 \%$, sensitivity would increase from 60 to $99 \%$. On the other hand, specificity would decrease from 97 to $86 \%$.

Our combined index had the advantage of enhancing sensitivity without losing specificity. It was designed in the clinical context of diabetic polyneuropathy, and all parameters were affected in a similar way. The most sensitive parameters were MCV-P and ASP-S, as previously reported by Dyck et al. ${ }^{10}$, but we could not demonstrate significant differences between the parameters due to the small number of patients. Our combined index is possibly not suited for the diagnosis of other types of polyneuropathy, such as vitamin deficiency or Guillain-Barré syndrome, because these parameters are not affected in the same way. Dyck et al. have shown that composite scores were less sensitive than isolated motor conduction parameters in patients with multifocal motor neuropathy ${ }^{11}$.

Combined nerve conduction indexes, also known as "composite nerve conduction scores", have been used successfully before $\mathrm{e}^{1,4,7,8,10-13}$. Perhaps the most popular one is the combined sensory index for carpal tunnel syndrome developed by Robinson et al., which showed higher sensitivity when compared to isolated sensory latencies differences ${ }^{8}$. Even so, combined indexes have not achieved wide acceptance, probably because they need some post-processing calculation and require well-established normative values.

A combined index provides better distinction between healthy and diseased subjects through the addition of multiple trends from each individual test ${ }^{8}$. A combined index should also be more reproducible, since it minimizes random experimental errors from isolated parameters, although we cannot demonstrate that with the present study. However, other studies have shown this effect $t^{4,7,11,14}$. A single parameter that is more reproducible and more representative is better suited for patient follow-up or for longitudinal studies, including clinical trials ${ }^{6}$.

There is some discussion regarding which is the best mathematical approach for normative values: normal deviations or percentiles ${ }^{5,15}$. Z-scores assume a Gaussian distribution for the normative data while percentiles do not ${ }^{15}$. On the other hand, percentiles require a larger normative sample ${ }^{15}$ and do not provide information on "how abnormal" is the parameter, since they cannot see beyond percentiles 0 and 100 . One great advantage of Z-scores is that they can be averaged while percentiles cannot. Percentiles have to be converted into standard deviations before they can be averaged ${ }^{11}$, which is fine when dealing with values in the intermediate range, but may be inaccurate when dealing with abnormal values.

A troublesome problem about averaging Z-scores is what to do with unobtainable parameters. We chose a conservative approach and excluded unobtainable parameters from 
the averaged Z-score. This excluded the most abnormal parameters from the analysis. Dyck et al. recommended a different approach: they would divide the averaged Z-score by the proportion of parameters used, increasing absolute obtained values $^{11}$. In other words, if only 8 out of 10 parameters were available, they would divide the averaged Z-score by 0.8 . Yet another approach was suggested by Joe Jabre (personal communication): he would arbitrarily attribute a Z-score value of -4.0 to unobtainable parameters. Specific abnormal values can also be attributed to unobtainable parameters ${ }^{4,13}$, such as $1 \mu \mathrm{V}$ for ASP-S or $30 \mathrm{~m} / \mathrm{s}$ for MCV-P. All options presented here are arbitrary and cannot be established in control subjects. The use of any of these approaches would actually increase the sensitivity of the combined index, but not of the other isolated parameters.

Our study has some methodological problems. It is a retrospective study, and we had limited clinical data from the subjects. Symptoms alone have poor diagnostic accuracy in predicting the presence of polyneuropathy ${ }^{2}$. It would be better to include clinical exam details in order to obtain more objective data confirming the presence of polyneuropathy, but this was not available in all subjects. Besides that, patients with burning feet due to small fiber neuropathy are not expected to have abnormalities in nerve conduction studies. This could explain the low sensitivity of isolated parameters, but it also affected the combined index in the same way. Subjects older than 65 were excluded despite the fact that polyneuropathy is more common in older patients. This was done because there is a higher chance of idiopathic polyneuropathy in this age group not related to diabetes mellitus. It also enhances the chance of unobtainable ASP-S or ASP-P with no clinical significance.

Our control group was not composed by healthy subjects. This could actually be an advantage, since healthy subjects are not referred for neurophysiological evaluation, and lumbosacral radiculopathy is an important differential diagnosis. However, patients with L5 radiculopathy may occasionally show abnormalities in MCV-P or even in ASP-P, and patients with spinal stenosis could have abnormal FW-T (although subjects with bilateral symptoms were not included), which means that specificity of nerve conduction studies could be even higher in normal subjects. The groups were also not comparable in terms of age or sex distribution, although Z-scores were corrected by age. Better designed prospective studies are required to confirm the utility of our combined index for the diagnosis of diabetic polyneuropathy.

We believe that combining several parameters of nerve conduction studies into a single index is a rational approach. A single combined index is more representative and more reproducible; it is also easier to interpret and has higher sensitivity without loss of specificity. Our normative values and regression equations for calculation of the combined index are provided here and can be easily replicated. It may seem complicated at first glance, but this is not a problem in the age of modern computers.

\section{References}

1. Feki I, Lefaucheur JP. Correlation between nerve conduction studies and clinical scores in diabetic neuropathy. Muscle Nerve 2001;24:555-558.

2. England JD, Gronseth GS, Franklin G, et al. Distal symmetric polyneuropathy: a definition for clinical research: report of the American Academy of Neurology, the American Association of Electrodiagnostic Medicine, and the American Academy of Physical Medicine and Rehabilitation. Neurology 2005;64:199-207.

3. Dyck PJ, Overland CJ, Low PA, et al. Signs and symptoms versus nerve conductionstudies to diagnose diabetic sensorimotor polyneuropathy: Cl vs. NPhys trial. Muscle Nerve 2010;42:157-164.

4. Solders G,AnderssonT,BorinY,BrandtL, PerssonA. Electroneurography index: a standardized method to assess peripheral nerve function in patients with polyneuropathy. Muscle Nerve 1993;16:941-946.

5. Peng L, Wuu J, Benatar M. Developing reference data for nerve conduction studies: an application of quantile regression. Muscle Nerve 2009;40:763-771.

6. Dyck PJ, O'Brien PC, Litchy WJ, Harper CM, Daube JR, Dyck PJB. Use of percentiles and normal deviates to express nerve conduction and other test abnormalities. Muscle Nerve 2001;24:307-370.

7. Tacconi P, Manca D, Tamburini G, et al. Electroneurography index based on nerve conduction study data: method and findings in normal subjects. Muscle Nerve 2004;29:89-96.
8. Robinson LR, Micklesen PJ, Wang L. Strategies for analyzing nerve conduction data: superiority of a summary index over single tests. Muscle Nerve 1998;21:1166-1171.

9. Heise CO, Toledo SM. Combined nerve conduction index for diagnosis of diabetic polyneuropathy. Muscle Nerve 2005;32:386.

10. Dyck PJ, Carter RE, Litchy WJ. Modeling nerve conduction criteria for diagnosis of diabetic polyneuropathy. Muscle Nerve 2011;44:340345 .

11. Dyck PJ, Litchy WJ, Daube JR, et al. Individual attributes versus composite scores of nerve conduction abnormality: sensitivity, reproducibility, and concordance with impairment. Muscle Nerve 2003;27:202-210

12. Jabre JF, Sato L. The expression of electrophysiologic data as mean related values. Muscle Nerve 1990;13:861-862.

13. Wennberg A. A neuropathy index based on sensory-motor electroneurography (ENeG). Acta Neurol Scand 1984;70:S107-S111.

14. Lew HL, Wang L, Robinson LR. Test-retest reliability of combined sensory index: implications for diagnosing carpal tunnel syndrome. Muscle Nerve 2000;23:1261-1264.

15. Robinson LR, Temkin NR, Fujimoto WY, Stolov WC. Effect of statistical methodology on normal limits in nerve conduction studies. Muscle Nerve 1991;14:1084-1090 